\title{
Erratum: Matrix product state formulation of frequency-space dynamics at finite temperatures [Phys. Rev. B 90, 060406(R) (2014)]
}

\author{
Alexander C. Tiegel, Salvatore R. Manmana, Thomas Pruschke, ${ }^{*}$ and Andreas Honecker \\ (Received 19 October 2016; published 16 November 2016)
}

\section{DOI: 10.1103/PhysRevB.94.179908}

The Gaussian resolution broadening $\eta$ of the zero-temperature spectral functions shown in Figs. 2(a), 2(b), and 3 of our original paper is frequency dependent and therefore not uniform as incorrectly stated. In this Erratum, we correct the resolution of these zero-temperature results by displaying them at a uniform broadening for all frequencies. Most importantly, the resolution of the spectral functions at both $T=0$ and $T>0$ are now the same in the revised Figs. 2 and 3 presented here.

The reason is that, besides the order $N$ of the Chebyshev expansions and the bandwidth $W$, the resolution of the spectral function also depends on the frequency $\omega[1]$,

$$
\eta(\omega)=\frac{\pi}{N} \frac{W}{2 W^{\prime}} \sqrt{1-\omega^{\prime 2}}
$$

Here $\omega^{\prime}$ denotes the linearly rescaled frequency which is a function of $\omega$. This dependence can be neglected for the studied finite-temperature spectral functions since within the Liouville-space formulation $\left|\omega^{\prime}\right| \ll 1$. However, at $T=0$ the linear rescaling maps the support of the spectral function onto the lower part of the interval $\left[-W^{\prime}, W^{\prime}\right]$, where $\omega^{\prime}$ is only slightly larger than -1 . Thus, the broadening $\eta(\omega)$ varies strongly as a function of frequency. This has been erroneously ignored by specifying a uniform broadening $\eta$ for the zero-temperature results in Figs. 2(a), 2(b), and 3 in our original paper. In fact, the $T=0$ resolution in the original paper is better than stated there, especially towards low frequency.

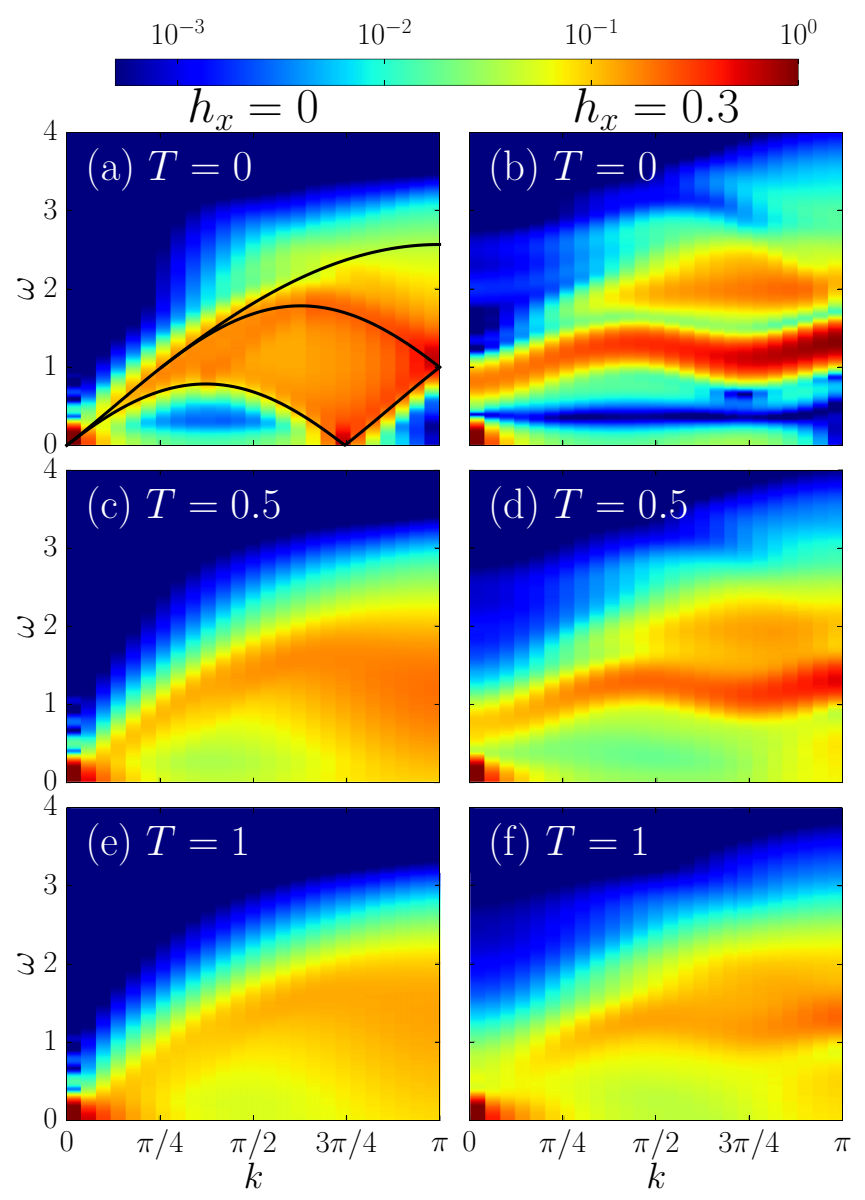

FIG. 2. Longitudinal spin structure factor $S_{z z}(k, \omega)$ of an isotropic Heisenberg chain $(J=1)$ in a magnetic field of $h_{z}=1$ obtained by the Chebyshev matrix product state (MPS) method with approximate Gaussian broadening $\eta$. Left column: $h_{x}=0(\eta \approx 0.13)$. Right column: $h_{x}=0.3(\eta \approx 0.14)$.

\footnotetext{
${ }^{*}$ Deceased.
} 


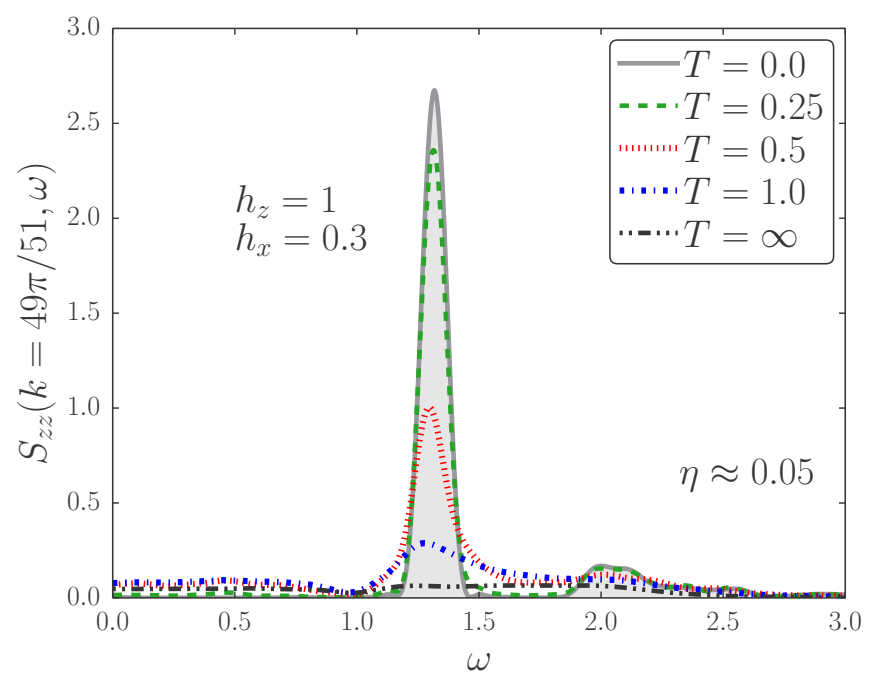

FIG. 3. MPS results for the frequency dependence of $S_{z z}(k, \omega)$ at $k=49 \pi / 51$ of an isotropic Heisenberg chain $(J=1)$ with $L=50$ sites in a uniform magnetic field of $h_{z}=1$ and an additional staggered field $h_{x}=0.3$ at temperatures of $T=0,0.25,0.5,1$, and $\infty$.

The revised Fig. 2 allows for a direct comparison between $T=0$ and $T>0$ results by choosing the same broadening for all frequencies. In order to introduce a frequency-independent broadening at $T=0$, the expansion order $N$ of the respective spectral functions is adapted as a function of frequency.

The results in the revised Fig. 3 have been obtained at an enhanced resolution $(\eta \approx 0.05$ instead of 0.14 ) in comparison to the original paper. The corrected resolution broadening of the zero-temperature spectral function is now frequency independent and corresponds with that of the finite-temperature results. Note that, after this correction, there is only slight thermal line-shape broadening of the main feature between $T=0$ and $T=0.25$ in the revised Fig. 3, whereas it is still present at higher $T$. The correction of the zero-temperature resolution has no further impact on the main results and conclusions of our paper.

[1] A. Weiße, G. Wellein, A. Alvermann, and H. Fehske, Rev. Mod. Phys. 78, 275 (2006). 